\title{
Science Writing Checklist
}

by Daniel M. Zuckerman (Oregon Health \& Science University) with helpful edits from E. Lyman and A. Mamonov, Jan 2010 at University of Pittsburgh; minor revision made Sep 2021 with input from L. Chong

General

$\square$ Does each paragraph focus on a single idea or point which is introduced/summarized in the paragraph's first sentence? A paragraph is like a mini-essay.

$\square$ Is the flow of logic clear from paragraph to paragraph? From your draft, you should be able to (re)write the outline of the paper - in fact, just from the first sentences of the paragraphs. Check this.

$\square \quad$ Did you repeat key points in several sections to emphasize them?

․ Did you spend a lot more time on logic and clarity than grammar and sentence structure? Nevertheless, avoid complicated sentences.

Abstract

Does the abstract avoid distracting technical details?

․ Is it clear from the abstract why the work is new and worthy of publication?

Introduction

$\square \quad$ Did you clearly explain the reason why the work was done - the existing problem?

ㅁ Did you clearly and briefly explain what you did to make progress - what's new?

․ Did you cite pertinent work done before? Is your list inclusive - demographically? of people you may not like? Citations affect careers, and we all have our biases.

$\square$ Did you read the introductions of several related papers to be sure you explained the ideas properly and cited the important work?

Methods

Did you remind your readers why a new/old method was used? You can write a mini-introduction for the Methods section.

$\square$ Did you provide enough information so a reader could exactly reproduce your results? The whole procedure should be outlined, even if some details must be found in other work or Supplemental Information.

$\underline{\text { Results }}$

Did you make sure the main results are not buried? Again, use mini-introductions.

$\square$ Did you save commentary and speculation for the Discussion section?

\section{Discussion}

Did you clearly explain what's new, as compared to previous work?

$\square$ Did you avoid repeating details from the Results section? Re-presenting the gist is a good idea.

$\square \quad$ Did you admit the limitations of your work?

$\square$ Did you describe future applications, improvements, and generalizations?

Conclusions

$\square$ Could a reader in a rush read just the Conclusions and learn just about everything (including acronyms)?

$\square \quad$ Did you avoid exaggeration and let the data speak for itself?

Did you acknowledge everyone who helped, including funding agencies?

\section{$\underline{\text { Figures }}$}

$\square$ Do figure titles describe the main point of each figure?

Have you put labels/arrows in the graphic to minimize effort for the reader?

Wait!

․ Did you go back to the 'General' section above and double-check those paragraphs and logic-even in the Results section? And is every paragraph in the right section?

$\square \quad$ Did you make several revisions of the entire manuscript (after completing a first draft)?

$\square \quad$ Did you check journal-specific formatting - section order; figures; references? 